\title{
Gene transfer using liposome-complexed adenovirus seems to overcome limitations due to coxsackievirus and adenovirus receptor-deficiency of cancer cells, both in vitro and in vivo
}

\author{
Sang-Young $\operatorname{Han}^{1 *}$, Yoon-Jong Lee ${ }^{2 *}$, \\ Haeng-Im Jung ${ }^{3}$, Sung-Wook Lee ${ }^{1}$, Sue-Jeong Lim ${ }^{4}$, \\ Seung-Hee Hong ${ }^{2,6}$ and Jin-Sook Jeong ${ }^{5,6}$ \\ ${ }^{1}$ Department of Internal Medicine \\ Dong-A University College of Medicine \\ Busan 602-714, Korea \\ ${ }^{2}$ Korea Human Resource Development \\ Institute for Health and Welfare \\ Seoul 122-701, Korea \\ ${ }^{3}$ Department of Internal Medicine \\ Yonsei University College of Medicine \\ Seoul 120-752, Korea \\ ${ }^{4}$ Department of Bioscience and Biotechnology \\ Sejong University, Seoul 143-747, Korea \\ ${ }^{5}$ Department of Pathology and \\ Medical Research Center for Cancer Molecular Therapy \\ Dong-A University College of Medicine \\ Busan 602-714, Korea \\ ${ }^{6}$ Corresponding authors: Tel, 82-51-240-2921; \\ Fax, 82-51-247-2903; E-mail, jsjung1 @ dau.ac.kr (J.S. Jeong) \\ Tel, 82-2-3156-1164; Fax, 82-2-3156-1189; \\ E-mail, hsh325@paran.com (S.H. Hong) \\ *These authors contributed equally to this work. \\ DOI 10.3858/emm.2008.40.4.427
}

Accepted 24 April 2008

Abbreviations: Ad, adenovirus; CAR, coxsackievirus and adenovirus receptor; DOPE, 1,2-dioleoyl-3-phosphatidylethanolamine; DOTAP, dioleoyltrimethylaminopropane; GFP, green fluorescent protein; $\mathrm{MOI}$, multiplicity of infection; luc, luciferase; PEGPE, 1,2-distearoylsn-glycero-3-phosphoethanolamine- $\mathrm{N}$-[methoxy(poly-ethylene glycol)-2000

\begin{abstract}
Use of adenoviruses as vehicle for gene therapy requires that target cells express appropriate receptors such as coxsakievirus and adenovirus receptor (CAR). We show here that CAR-deficiency in cancer cells, that limits adenoviral gene delivery, can be overcome by using adenovirus complexed with the liposome, Ad-PEGPE [1,2-distearoyl-sn-glycero-3-phosphoethanolamine-N-[methoxy(poly-ethylene glycol)-2000]. We first confirmed that CT-26 mouse colon cancer cells are
\end{abstract}

deficient in CAR by RT-PCR, and then showed that CT-26 cells infected with Ad-GFP/PEGPE exhibited highly enhanced expression of green fluorescent protein (GFP), compared with those infected with Ad-GFP. GFP expression depends on the dose of liposome and adenovirus. Luciferase expression in livers treated with Ad-luc/PEGPE was about 1,000-fold less than those infected with Ad-luc. In a liver metastasis mouse tumor model developed by intrasplenic injection of CT-26 cells, luciferase expression following i.v. injection of Ad-luc/PEGPE was significantly higher in tumors than in adjacent non-neoplastic liver. Following systemic administration of Ad-GFP/PEGPE, GFP expression increased in tumors more than in adjacent liver while the reverse was true following administration of Ad-GFP. In the latter case, GFP expression was higher in liver than in tumors. This study demonstrates that systemic delivery of PEGPE-adenovirus complex is an effective tool of adenoviral delivery as it overcomes limitation due to CAR deficiency of target cells while reducing hepatic uptake and enhancing adenoviral gene expression in tumors.

Keywords: adenovirus; CXADR protein, human; gene therapy; gene transfer techniques; liposomes; models, animal

\section{Introduction}

Recombinant adenoviruses are one of the most useful vectors for gene transfer, and particularly suitable for cancer gene therapy (Kozarsky and Wilson, 1993). They are capable of infecting a large range of cell types in all stages of cell division (St George, 2003). Because the genetic and biological characteristics of Ad serotype 5 (Ad5) are well understood, Ad5 which belongs to subgroup $C$, is the most common adenovirus used. Ad5 requires coxsackievirus and adenovirus receptor (CAR) on target cells as a primary receptor for infection (Bergelson et al., 1997; Tomko et al., 1997), and the transduction efficiency of the Ad5-based vectors closely correlate with the cell-surface density of CAR (Hemmi et al., 1998; Li et al., 1999). Biodistribution studies of systemically administered adenovirus have revealed that adenovirus 
preferentially accumulates in the livers of rodents and non-human primates (Huard et al., 1995; Sullivan et al., 1997), presumably via CAR on the hepatocytes.

An important issue confronting gene transfer by adenovirus is inefficient transduction to target neoplastic (Miller et al., 1998; Li et al., 1999), or non-neoplastic cells (Nalbantoglu et al., 1999; Shayakhmetov et al., 2000), because of insufficient CAR expression. Thus the level of CAR expression may be a limiting factor for the transduction efficiency achievable with adenovirus. Other limitations of the use of adenoviral vectors include toxicity and readministration.

Complexing adenovirus with cationic liposomes or polymers, has been shown to be effective in increasing adenoviral transgene expression by facilitating adenovirus binding to the cell surface, particularly on CAR deficient cells (Fasbender et al., 1997; Lee et al., 2000). Liposome complexation also resulted in reduced immunogenicity of adenovirus (Chillon et al., 1998; Yotnda et al., 2002).

In the present study, we attempted to overcome the reduced gene expression of adenovirus caused by CAR deficiency in CAR-negative cancer cells in vitro as well as in vivo. We first confirmed that complexing adenoviral vectors with liposomes provided a valuable approach for gene therapy in vtro in CAR-negative cancer cells. We then extended this approach to an in vivo study, in a metastatic liver tumor model. This study suggests the feasibility of this approach in the gene therapy of human cancers in general.

\section{Materials and Methods}

\section{Cell lines and reagents}

CT-26 (mouse colon cancer cell line), Hepa1-6 (mouse hepatoma cell line), NIH3T3 (mouse fibroblast cell line), and B16F10 (mouse melanoma cell line) were used in these experiments. These cell lines were purchased from ATCC (American Type Culture Collection) and cultured in DMEM (GIBCO, Gaithersburg, MD) supplemented with $10 \%$ FBS (Invitrogen, Carlsbad, CA) and $100 \mathrm{U} / \mathrm{ml}$ penicillin/streptomycin and incubated in humidified incubator at $5 \% \mathrm{CO}_{2}$.

Dioleoyltrimethylaminopropane (DOTAP), 1,2dioleoyl-3-phosphatidylethanolamine (DOPE), and 1,2-distearoyl-sn-glycero-3-phosphoethanolamine$\mathrm{N}$-[methoxy(poly-ethylene glycol)-2000 (PEGPE) were purchased from Avanti Polar Lipids (Alabaster, $A L)$. Lipofectin was purchased from Gibco BRL (Life Technologies, Gaithersburg, MD).

The recombinant adenovirus, Ad-GFP, was pro- pagated in a permissive 293 cell line and purified by centrifugation in cesium chloride gradients. One plaque forming unit (PFU) was defined as 100 virus particles. The virus stock was kept frozen at $80^{\circ} \mathrm{C}$ until use.

\section{Preparation of cationic liposome}

Cationic liposomes were prepared using an extrusion method. Briefly, DOTAP and DOPE with or without PEGPE were mixed at a molar ratio of $1: 1: 0.01$ equimolar amounts of DOTAP to DOPE mixed with or without $0.01 \mathrm{M}$ of PEGPE in chloroform. The mixture was dried at $40^{\circ} \mathrm{C}$ in a rotary evaporator. The dried lipid film was hydrated with $5 \%$ dextrose dissolved in distilled water. After incubation for $30 \mathrm{~min}$ at room temperature or overnight at $4{ }^{\circ} \mathrm{C}$, the liposome solution was extruded (Lipofast-Basic, Avestin, Canada) through a 100 $\mathrm{nm}$ polycarbonate membrane. The extrusion step was repeated until a homogeneous batch of liposomes of 100-150 nm mean particle size was obtained. The particle size was monitored by dynamic light scattering method using an electrophoretic light scattering spectrophotometer (ELS-8000, OTSUKA Electronics, Japan). The liposomes were stored at $4^{\circ} \mathrm{C}$ until use.

\section{RT-PCR analysis}

Total RNAs of the cell lines were isolated using TRIzol reagent (Promega Corporation, Madison, WI) according to the manufacture's protocol. To remove contaminated DNA, the RNA preparations were treated with $1 \mathrm{U}$ of DNase I (Promega Corporation, Madison, $\mathrm{WI})$ for $30 \mathrm{~min}$ at $37^{\circ} \mathrm{C}$.

cDNAs were synthesized from the purified RNAs using Omniscript reverse transcriptase (QIAGEN $\mathrm{GmbH}$, Hilden, Germany) with $250 \mathrm{ng}$ of random primer (Invitrogen, Carlsbad, CA). The cDNAs were amplified with mouse CAR primers (5'-TTCCTGCTGACCGTTCTTG-3' and 5'-GTCTAGTCGCAGCATACAG-3'), or mouse $\alpha v$ integrin primers (5'CAAGCTCACTCCCATCAC-3' and 5'-GGGTGTCTTGATTCTCAAAGGG-3') or mouse GAPDH primers (5'-TGATGACATCAAGAAGGTGGTGAAG-3' and 5'-TCCTTGGAGGCCATGTGGGCCAT-3'). The amplification reaction was carried out with cDNA for 30 cycles $\left(95^{\circ} \mathrm{C}, 30 \mathrm{~s} ; 56^{\circ} \mathrm{C}, 30 \mathrm{~s} ; 72^{\circ} \mathrm{C}, 30 \mathrm{~s}\right)$, and the PCR products were fractionated on $1.5 \%$ agarose gels.

\section{Adenovirus infection}

Cells were seeded in 12-well plates at $10^{5}$ cells/well and used when $70-80 \%$ of cells were confluent. 
Complexes of adenovirus and cationic liposomes were made by mixing Ad-GFP and liposomes suspended in $100 \mu$ OptiMEM (Gibco, Life Technologies, Gaithersburg, MD), followed by incubation for $20 \mathrm{~min}$ at room temperature. Cells were washed with PBS, and added to the virus-liposome complexes and $400 \mu \mathrm{l}$ medium. After $4 \mathrm{~h}$ incubation in a $\mathrm{CO}_{2}$ incubator at $37^{\circ} \mathrm{C}$, the cells were washed with PBS to remove the complexes, and $1 \mathrm{ml}$ of fresh medium was added. The cells were then incubated for an additional $24 \mathrm{~h}$ before assessing GFP expression.

\section{In vitro gene expression}

The cells were harvested, washed twice with PBS, and fixed with $1 \%$ paraformaldehyde for $30 \mathrm{~min}$ at $4^{\circ} \mathrm{C}$. After brief centrifugation, the supernatant was discarded and the fixed cells were resuspended in PBS. The expression of GFP in suspended cells was directly determined using a FACS (Becton Dickinson, San Jose, CA). For obtaining the GFP signal with fluorescence emission centered at 530 $\mathrm{nm}, 5000$ fluorescent events per sample were acquired using a 530/15 band pass filter.

\section{Animals}

Six to eight week old male BALB/c mice were obtained from Orient (Seoul, Korea) and housed in an animal facility (Korea Food and Drug Administration: Unit Number-000996), in cages under a strict light cycle (lights on at 06:00 $\mathrm{h}$ and off at 18:00 h), in accordance with AAALAC International Animal Care policy.

\section{Liver metastasis tumor model}

We established a mouse liver metastasis tumor model by intrasplenic injection into the animals, of CAR-deficient CT-26 colon cancer cells essentially as desribed (Giavazzi et al., 1986), with some modifications. Briefly, a small longitudinal left upper frank incision was made to make the spleen visible, and $1 \times 10^{6}$ of CT-26 cells in $50 \mu$ of PBS were injected below the splenic capsule with a 29-gauge needle. After removal of the needle, the injection site was pressed with an aseptic cotton sponge for several minutes, the spleen returned into abdominal cavity, and the peritoneum and abdominal wall were sutured with silk. The animals developed metastatic liver tumors within 8-12 days, easily detectable by gross inspection.

\section{In vivo animal study}

To determine the transgene expression of viruses and virus-liposome complexes in normal and liver tumor-bearing mice, random groups of mice were administered, respectively with PBS, $1 \times 10^{11} \mathrm{vp} / 100$ $\mu l$ adenovirus, or liposome-complexed adenovirus via tail vein. For luciferase expression assay, normal mice were treated with PBS $(n=5)$, Ad-luc $(n=5)$, or Ad-luc/PEGPE $(n=5)$ and sacrificed after $24 \mathrm{~h}$ injection. Mice with intrahepatic metastatic tumors were treated with Ad-luc $(n=4)$ and Ad-luc/PEGPE $(n=4)$. Normal liver and tumor nodules were harvested and homogenized in lysis buffer (Promega Corporation, Madison, WI). Luciferase expression was measured using the Luciferase Assay Reagent (Promega Corporation, Madison, WI) according to manufacturer's instruction and luciferase activity was calculated as relative light units (RLU)/well.

For assessing in situ tissue distribution of adenovirus and liposome-complexed adenovirus, the tumor-bearing mice were treated with $1 \times 10^{11}$ $\mathrm{vp} / 100 \mu \mathrm{l}$ of Ad-GFP $(n=4)$ or Ad-GFP/PEGPE $(n$ $=4)$ through tail veins. After $24 \mathrm{~h}$, the mice were

A

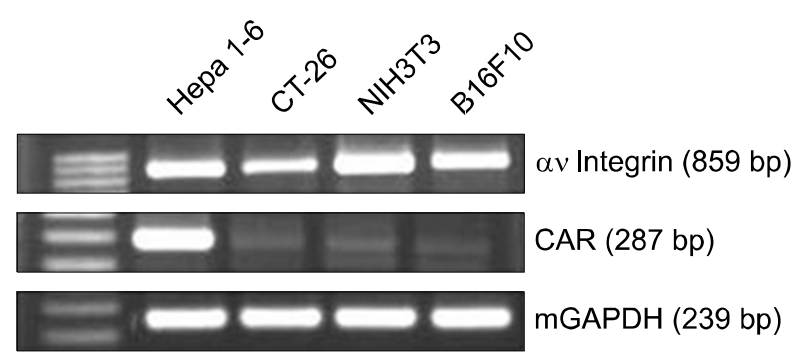

B

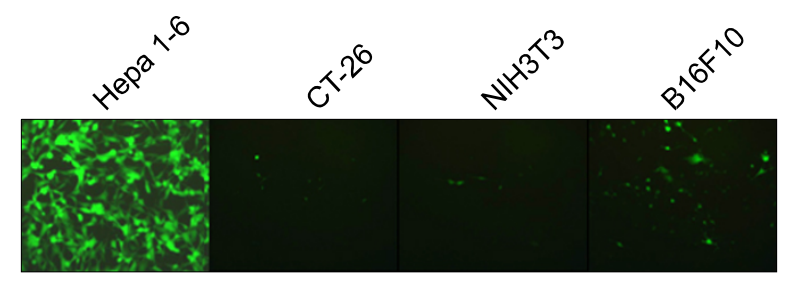

Figure 1. Expression of CAR and $\alpha v$ integrin in cancer cell lines after Ad-GFP treatment. (A) Expression levels of CAR and av integrin mRNA transcripts in mouse cancer cell lines. Total RNAs were isolated from each cell lines and RT-PCR was performed with mouse GAPDH as a control. PCR products were separated on $1.2 \%$ agarose gels and stained with ethidium bromide. (B) Adenoviral gene expression in CAR-positive and -negative cell lines. Mouse cancer cells were seeded in six-well plates and infected with $30 \mathrm{MOI}$ of Ad-GFP to Hepa1-6 cells or $200 \mathrm{MOI}$ of Ad-GFP to CT-26, NIH3T3, and B16F10 cells. After 1 day, GFP expression in each cells was determined at $40 \times$ magnification taken using fluorescent microscope. 
sacrificed and their livers bearing tumor nodules were removed, sectioned, and representatives were frozen in cryoprotective medium (OCT; Sakura Fineteck, Torrance, CA). Eight- $\mu \mathrm{m}$ frozen sections were fixed with $4 \%$ paraformaldehide in $100 \mathrm{mM}$ PBS ( $\mathrm{pH} 7.4$ ) for $10 \mathrm{~min}$ at room temperature. The sections were washed twice with PBS, and mounted. Fluorescence was observed by Olympus fluorescent microscope. The slides were then, stained with hematoxylin and eosin, and the histologic features were observed under light microscope.

\section{Results}

\section{Expression level of CAR in mouse cancer cell lines}

Infection by adenovirus occurs in two distinct steps: binding to CAR on cell surface, and internalization by $\alpha v$ integrin. We examined the expressions of both CAR and $\alpha v$ integrin by RT-PCR analysis in several mouse cancer cell lines. We found that both CAR and $\alpha v$ integrin mRNA levels were high in Hepa1-6 cells (Figure 1A). CAR was not expressed in CT-26, NIH3T3, and B16F10 cell lines. $\alpha v$ intergrin was expressed in all cell lines although at a slightly low level in CT-26 cells.

In order to assess CAR-dependence of transgene expression of adenovirus, various mouse cancer cell lines were infected with Ad-GFP and GFP expression was examined. In CAR and $\alpha v$ integrin expressing Hepa1-6 cells, adenoviral GFP was expressed in about $90 \%$ of cells at 30 multiplicity of infection (MOI) of adenovirus (Figure 1B). In contrast, adenoviral GFP expression was not observed at $200 \mathrm{MOI}$ of adenovirus in CAR-negative cell lines: CT-26, NIH3T3, and B16F10 cells.

\section{Adenoviral gene expression in CAR deficient cells by liposome-complexed adenovirus depends on the dose of liposome and virus}

We demonstrated previously that addition of PEGPE enhances the stability and uptake of liposomecomplexed adenovirus in CAR deficient human cancer cell lines (Lee et al., 2004). Here we investigated the effect of liposome dose on the efficiency of liposome-complexed adenoviral gene transfer in CAR deficient CT-26 cell lines. We prepared cationic liposome (DOTAP:DOPE:PEGPE) and complexed it with adenovirus. For control, we used the commercially available liposome Lipofectin, which is a $1: 1$ liposome formulation of the cationic lipid N-[1-(2,3-dioleyloxy)propyl]-N,N,N-trimethylammonium chloride (DOTMA) and DOPE. Fixed amounts $\left(1 \times 10^{2} \mathrm{pfu} / \mu \mathrm{l}\right)$ of Ad-GFP were incubated with varying concentrations of liposome as used for CT-26 cell infections and adenoviral GFP expressions were measured by FACS. We found that adenoviral GFP expression in CT-26 cells increased with increasing concentrations of PEGPE liposome reaching a plateau $(52.96 \pm 2.92 \%)$ at $2.5 \mu \mathrm{l} / 1 \times$ $10^{2}$ pfu (Figure 2A). In contrast, adenoviral GFP expression did not increase with increasing concentration of Lipofectin. This shows that PEGPE liposome enhanced the transduction efficiency of
A

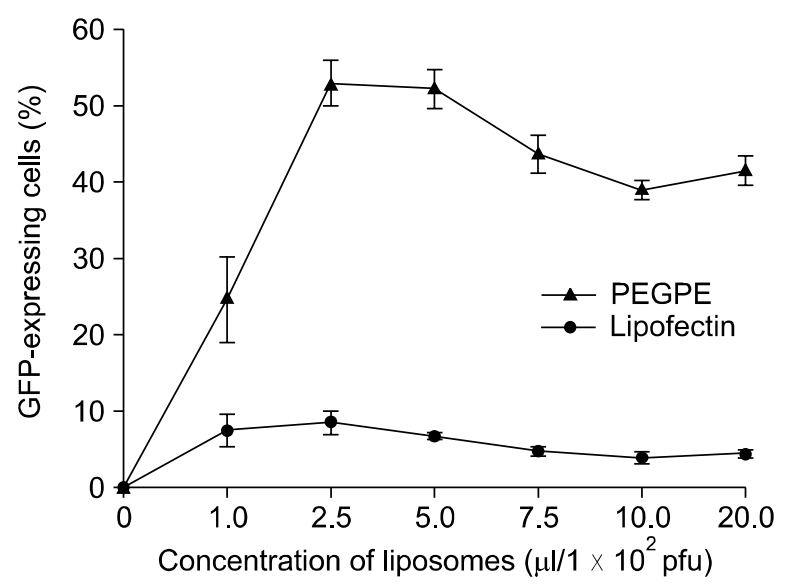

B

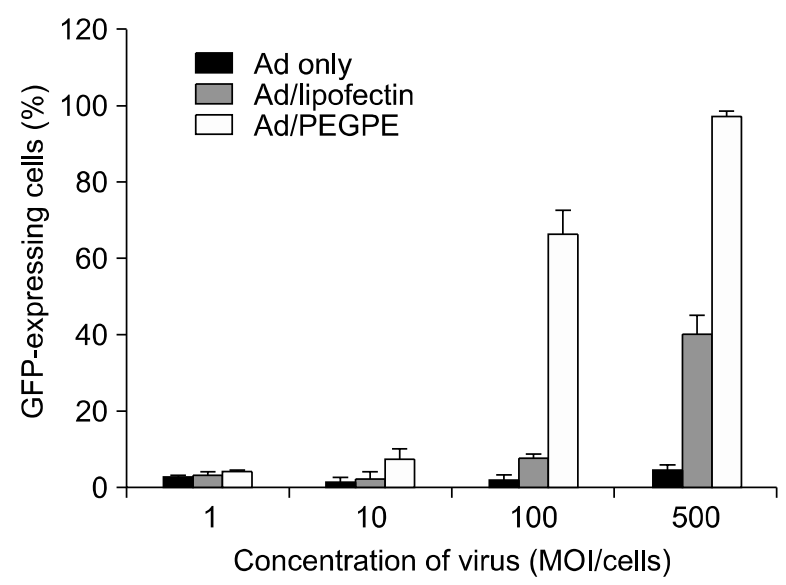

Figure 2. Effect of liposome dose and adenovirus concentration on efficacy of liposome-complexed adenoviral gene expression. (A) Effect of liposome dose on adenoviral gene expression in CT-26 cells. Varying amounts of PEGPE liposome were incubated with concentration of Ad-GFP $\left(1 \times 10^{2}\right.$ pfu/ $\mu$ l) for $30 \mathrm{~min}$ and then added to CT-26 cells. After $24 \mathrm{~h}$, the percentage of GFP expressing cells was determined by FACS analysis. The data represents mean \pm S.D. of triplicate experiments. (B) Effect of adenovirus concentration. Varying concentrations of Ad-GFP were incubated with PEGPE liposome at fixed liposome dose $(2.5 \mu \mathrm{l})$. The next experimental procedures were the same as $(\mathrm{A})$. 
adenovirus in a dose dependent manner.

Based on above data we chose the liposome dose of $2.5 \mu \mathrm{l}$, for assessing the effect of adenovirus on liposome-complexed adenoviral gene transfer in CT-26 cells. We infected CT-26 cells with various concentrations of PEGPE-complexed, Lipofectincomplexed, or naked adenovirus, and examined adenoviral GFP expression. As shown in Figure 2B, adenoviral GFP expression significantly increased when complexed with PEGPE $(3.82 \pm 0.65$ at 1 $\mathrm{MOI}, 7.15 \pm 2.74$ at $10 \mathrm{MOI}, 66.23 \pm 6.29$ at 100 $\mathrm{MOI}$, and $97.03 \pm 1.57 \%$ at $500 \mathrm{MOI}$ ). But, when complexed with Lipopectin, adenoviral GFP expression increased only slightly $(39.95 \pm 4.80 \%$ at $500 \mathrm{MOI}$ ) and the uncomplexed naked adenovirus did not influence GFP expression. Thus, it is clear that complexation with PEGPE leads to adenoviral gene expression in CAR deficient cancer cell lines in a manner dependent on the dose of both liposome and adenovirus.

\section{Tissue distribution of systemically administered liposome-complexed adenovirus}

To examine adenoviral gene expression in normal liver, we injected $B A L B / c$ mice with Ad-luc or Ad-luc/PEPGE $\left(1 \times 10^{11} \mathrm{vp} / \mathrm{mouse}\right)$ via tail veins and determined luciferase expression in liver. High level of luciferase expression was observed after Ad-luc administration (Figure 3A). In contrast, the administration of the same dose of Ad-luc/PEPGE reduced luciferase expression by a factor of 1000 , in normal liver. This also suggests that gene expression of liposome complexed adenovirus in the liver is reduced with consequent reduction of liver toxicity.

We then compared the expression of luciferase in the lungs, hearts, kidneys, and spleens of mice, intravenously administered with Ad-luc and Ad-luc/ PEGPE. As shown in Figure 3B, with Ad-luc, there was a preferential luciferase expression in the liver relative to the other tissues examined. In contrast, Ad-luc/PEGPE caused enhanced luciferase expression in all tissues examined. This indicates that transduction by systemic administration in vivo of Ad-luc/PEGPE was not specific to the liver tissue.

\section{In vivo expression of liposome-complexed adenoviral genes in liver metastasis tumor model}

To monitor adenoviral gene expression in metastatic liver tumors, we injected BALB/c mice with Ad-GFP or Ad-GFP/PEPGE $\left(1 \times 10^{11} \mathrm{vp} / \mathrm{mouse}\right)$ via tail vein and observed GFP expression in the livers. The animals injected with Ad-GFP showed markedly decreased fluorescence in metastatic tumors in comparison to adjacent non-neoplastic liver (Figure 4A, upper panel). In contrast, injection of the same dose of Ad-GFP/PEPGE, resulted in increased fluorescence in metastic tumors, which was higher than or similar to that seen in adjacent nonneoplastic liver (Figure 4A, low panel).

We also examined the expression of luciferase in metastatic liver tumors and adjacent nonneoplastic liver in animals injected with Ad-luc and Ad-luc/PEGPE. There was lower luciferase expression in metastatic tumors than in adjacent nonneoplastic livers, in animals treated with Ad-luc
A

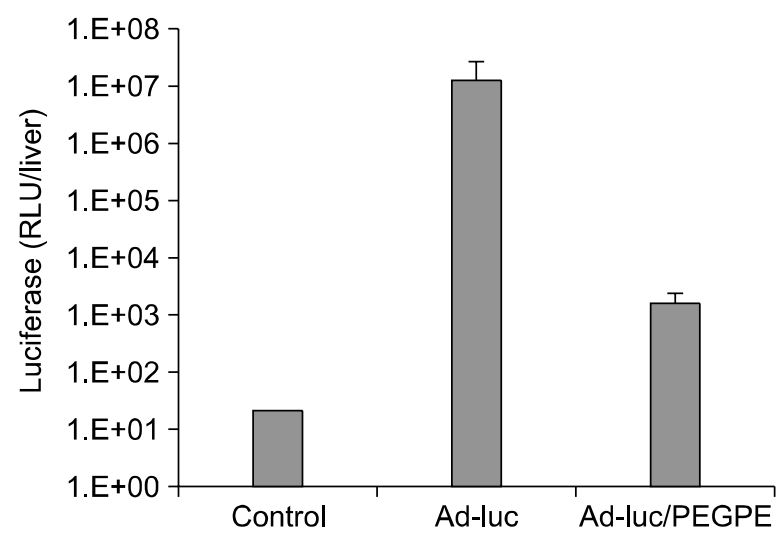

B

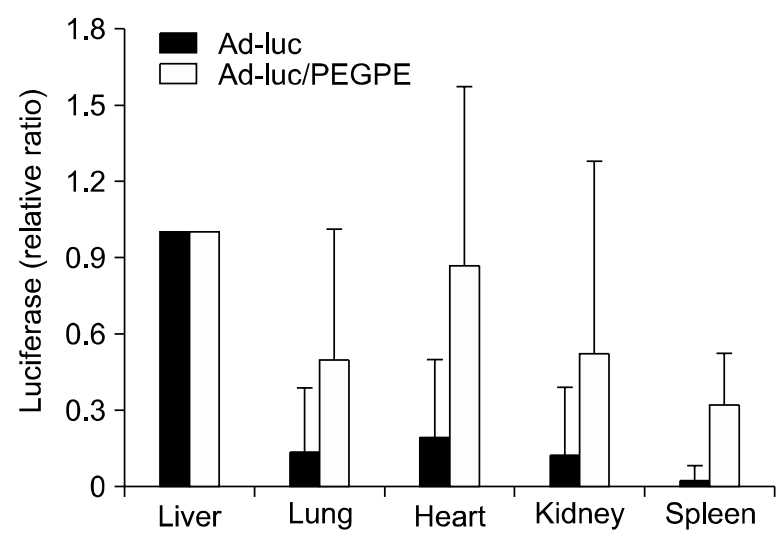

Figure 3. Tissue distribution of liposome-complexed adenovirus in normal mice after systemic administration. (A) Luciferase expression in liver following systemic administration of adenoviruses. $1 \times 10^{11} \mathrm{vp}$ of Ad-luc or Ad-luc/PEGPE was injected intravenously and transgene expression was measured after $24 \mathrm{~h}$. Liver was harvested, homogenized and then luciferase activity was measured using the kit according to the manufacturer's instructions. Each data represents the mean \pm S.D. $(n=5)$. (B) Tissue distribution of luciferase expression following systemic administration of adenoviruses. $1 \times 10^{11} \mathrm{vp}$ of Ad-luc or Ad-luc/PEGPE was intravenously injected into the Balb/c mice and luciferase expression was measured in each tissue. Luciferase activity in each tissue was calculated relative that in the liver. The data represent the mean \pm S.D. 
A

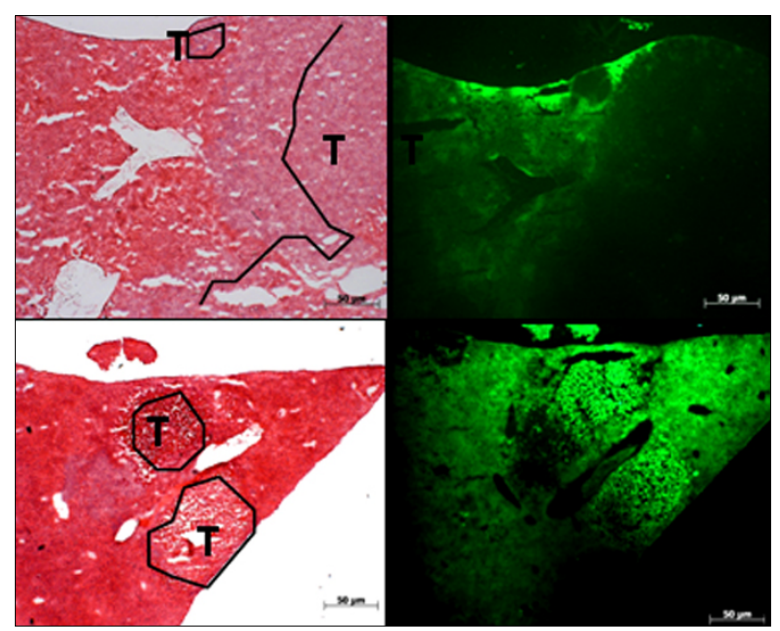

B

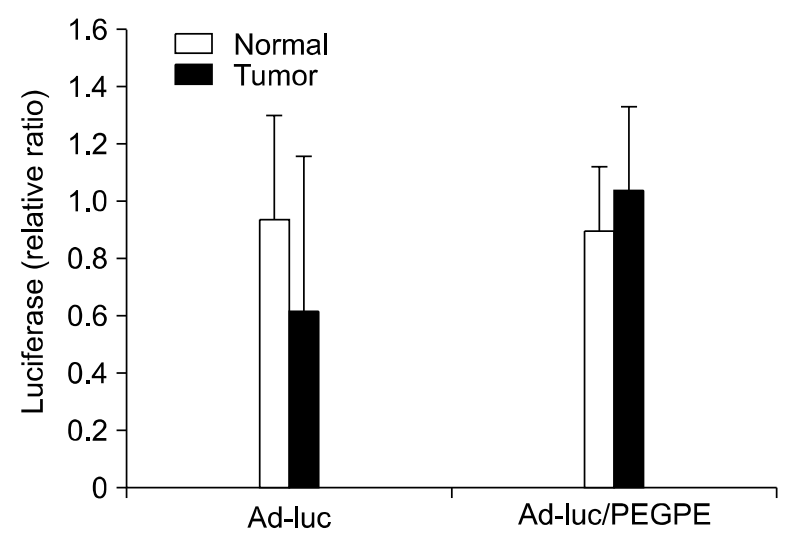

Figure 4. Expression of liposome-complexed adenovirus in liver metastasis tumor model. (A) GFP expression in intrahepatic tumors (T) and adjacent liver following systemic administration of adenoviruses. $1 \times 10^{11} \mathrm{vp}$ of Ad-GFP or Ad-GFP/PEGPE was injected intravenously and livers, including tumors were removed after $24 \mathrm{~h}$. Eight- $\mu \mathrm{m}$ frozen sections were prepared and mounted with cover glass. Fluorescence was observed by Olympus fluorescent microscope $(\times 40)$. After then, the slides were stained with hematoxylin and eosin, and observed under the light microscope $(\times 40)$. $(B)$ Relative ratio of luciferase expression of intrahepatic tumors to adjacent non-neoplastic liver following systemic administration of adenoviruses. $1 \times 10^{11}$ vp of Ad-luc or Ad-luc/PEGPE was intravenously injected and luciferase expression was measured in tumors and adjacent normal livers. Luciferase activity in tumors was calculated by relative to that in adjacent non-neoplastic liver. Data represents the mean \pm S.D.

(Figure 4B), in contrast to relatively higher luciferase expression in metastatic tumors than in adjacent non-neoplastic liver, following treatment with Ad-luc/PEGPE. These results indicate that systemically administered Ad-luc/PEGPE is efficiently transduced in intrahepatic colon cancer metastasis.

\section{Discussion}

Genetic alteration is known as the main cause of development and progression of cancer. Gene transfer is a potential tool to correct genetic alterations. Adenoviral vector-mediated gene manipulation is a potential approach (Song et al., 2007), but CAR-deficiency in some cancer cells prevents effective adenoviral infection of target cells. We previously found to enhance the stability and uptake of liposome-complexed adenovirus in CAR deficient human cancer cell lines (Lee et al., 2004). In this study we demonstrated that the problem of CAR-deficiency, can be overcome by complexing the adenovirus with PEGPE in vitro as well as in vivo in a mouse metastasis model we established, by injecting syngenetic mice with CAR-deficient CT-26 mouse colon cancer cells.

In CT-26 cells, liposome-complexed adenovirus mediated gene transfer was dependent on dose of liposome which plateaued at a dose of $2.5 \mu \mathrm{l} / 1 \times$ $10^{9} \mathrm{pfu}$ of liposome, and also the dose of the adenoviral vector which increased adenoviral GFP expression only slightly. Excess of liposome did not show transgenic expression in CT-26 cell culture. The high ratio of cations to adenovirus particles was considered to facilitate the aggregation of viral particles and decrease viral infection (Fasbender et al., 1997). An alternate possibility suggested is that higher doses of liposome are toxic to cells (Toyoda et al., 2001). We also observed that commercially available liposome, Lipopectin, was not effective in gene expression in agreement with a previous report (Byk et al., 1998).

Intratumoral injection of a complex of adenovirus and liposome led to enhanced adenoviral transduction compared to injecting just the adenovirus alone (Lee et al., 2000; Chae et al., 2005). Lipofectamin-mediated gene transfer was noted to be ineffective in liver-directed gene transfer in non-human primates (Sullivan et al., 1997). Our data show that hepatic uptake and luciferase expression mediated by liposome-complexed adenovirus decreased more markedly than Ad-luc mediated luciferase expression. Cations or polymers complexed with adenovirus were considered to interfere the interaction of adenovirus and CAR of liver cells. In addition, several reports provided evidence that immune responses are protected by readministration of liposome-complexed adenoviruses (Sullivan et al., 1997; Chillon et al., 1998; Yotnta et al., 2002). Liver-directed adenoviral gene 
transfer usually caused toxicity in CAR-abundant normal liver, immune responses and targeting of unwanted cells. We therefore attempted to confirm in vivo transgenic expression in animal models that especially mimic human fatal cancers.

We believe that the liver metastasis tumor model we established is similar to refractory human metastatic colon cancer. Intrasplenic injection of colon cancer cells which led to liver metastasis has proved useful for the study of the biology and therapy of the liver metastasis (Giavazzi et al., 1986). Our studies with this model revealed that liposome-complexed adenovirus mediated luciferase and GFP expression in the tumor bearing livers than in the adjacent non-neoplastic liver, and that adenovirus mediated luciferase expression mostly increased in non-neoplastic liver, and not in tumors. To our knowledge there are no previous reports of liposome-complexed adenovirus mediated gene expression in liver metastasis models. Further studies are needed to examine the efficacy of liposome-complexed adenovirus mediated gene transfer in CAR-deficient target cells.

In conclusion, this study demonstrates that limitations of gene therapy approaches involving the use of adenoviral vectors due to the CAR deficiency of target cells and liver toxicity can be overcome by using complexing the adenovirus with a liposome. Whether complexation of adenoviruses with liposomes provides a more effective adenoviral gene delivery system, needs to be established in clinical trials.

\section{Acknowledgement}

This paper was supported by the Dong-A University Research Fund in 2005.

\section{References}

Bergelson JM, Cunningham JA, Droguett G, Kurt-Jones EA, Krithivas A, Hong JS, Horwitz MS, Crowell RL, Finberg RW. Isolation of a common receptor for Coxsackie B viruses and adenoviruses 2 and 5. Science 1997;275:1320-3

Byk T, Haddada H, Vainchenker W, Louache F. Lipofectamine and related cationic lipids strongly improve adenoviral infection efficiency of primitive human hematopoietic cells. Human Gene Ther 1998;9:2493-502

Chae HY, Lee BW, Oh SH, Ahn YR, Chung JH, Min YK, Lee MS, Kim KW. Effective glycemic control achieved by transplanting non-viral cationic liposome-mediated VEGFtransfected islets in streptozotocin-induced diabetic mice. Exp Mol Med 2005;37:513-23

Chillön M, Lee JH, Fasbender A, Welsh MJ. Adenovirus complexed with polyethylene glycol and cationic lipid is shielded from neutralizing antibodies in vitro. Gene Ther

\section{8;5:995-1002}

Fasbender A, Zabner J, Chillőn M, Moninger TO, Puga AP, Davidson BL, Welsh MJ. Complexes of adenovirus with polycationic polymers and cationic lipids increase the efficiency of gene transfer in vitro and in vivo. J Biol Chem 1997;272:6479-89

Giavazzi R, Jessup JM, Campbell DE, Walker SM, Fidler IJ. Experimental nude mouse model of human colorectal cancer liver metastases. J Natl Cancer Inst 1986;77:1303-08

Hemmi S, Geertsen R, Mezzacasa A, Peter I, Dummer R. The presence of human coxsackievirus and adenovirus receptor is associated with efficient adenovirus-mediated transgene expression in human melanoma cell cultures. Hum Gene Ther 1998;9:2363-73

Huard J, Lochmuller H, Acsadi G, Jani A, Massie B, Karpati $G$. The route of administration is a major determinant of the transduction efficiency of rat tissues by adenoviral recombinants. Gene Ther 1995;2:107-15

Kozarsky KF, Wilson JM. Gene therapy: adenovirus vectors. Curr Opin Genet Dev 1993;3:499-503

Lee EM, Hong SH, Lee YJ, Kang YH, Choi KC, Choi SH, Kim IH, Lim SJ. Liposome-complexed adenoviral gene transfer in cancer cells expression various levels of coxsackievirus and adenovirus receptor. J Cancer Res Clin Oncol 2004; 130:169-77

Lee SG, Yoon SJ, Kim CD, Kim K, Lim DS, Yeom YI, Sung MW, Heo DS, Kim NK. Enhancement of adenoviral transducton with polycationic liposomes in vivo. Cancer Gene Ther 2000;7:1329-35

Li Y, Pong RC, Bergelson JM, Hall MC, Sagalowsky Al, Tseng CP, Wang Z, Hsieh JT. Loss of adenoviral receptor expression in human bladder cancer cells: a potential impact on the efficacy of gene therapy. Cancer Res 1999;59:325-30

Miller CR, Buchsbaum DJ, Reynolds PN, Douglas JT, Gillespie GY, Mayo MS, Raben D, Curiel DT. Differential susceptibility of primary and established human glioma cells to adenovirus infection: targeting via the epidermal growth factor receptor achieves fiber receptor-independent gene transfer. Cancer Res 1998;58:5738-48

Nalbantoglu J, Pari G, Karpati G, Holland PC. Expression of the primary coxsackie and adenovirus receptor is downregulated during skeletal muscle maturation and limits the efficacy of adenovirus-mediated gene delivery to muscle cells. Hum Gene Ther 1999;10:1009-19

Shayakhmetov DM, Papayannopoulou T, Stamatoyannopoulos $G$, Lieber $A$. Efficient gene transfer into human CD34(+) cells by a retargeted adenovirus vector. J Virol 2000; 74:2567-83

Song MS, Jeong JS, Cho KS, Lee SW. Gene expression responses in vivo by human telomerase reverse transcriptase (hTERT)-targeting trans-splicing ribozyme. Exp Mol Med 2007;39:722-32

St George JA. Gene therapy progress and prospects: adenoviral vectors. Gene Ther 2003;10:1135-41

Sullivan DE, Dash S, Du H, Hiramatsu N, Aydin F, Kolls J, Blanchard J, Baskin G., Gerber MA. Liver-directed gene 
transfer in non-human primates. Hum Gene Ther 1997;8: 1195-206

Tomko RP, Xu R, Philipson L. HCAR and MCAR: the human and mouse cellular receptors for subgroup $C$ adenoviruses and group B coxsackieviruses. Proc Natl Acad Sci USA 1997;94:3352-6

Toyoda K, Nakane H, Heistad DD. Cationic polymer and lipids augment adenovirus-mediated gene transfer to cerebral arteries in vivo. J Cereb Blood Flow Metab 2001; 21:1125-31

Yotnda P, Chen DH, Chiu W, Piedra PA, Davis A, Templeton NS, Brenner MK. Bilamellar cationic liposomes protect adenovectors from preexisting humoral immune responses. Mol Ther 2002;5:233-41 